\title{
Protocol Forms Set
}

National Cancer Institute

\section{Source}

National Cancer Institute. Protocol Forms Set. NCI Thesaurus. Code C41113.

Protocols, with Case Report Forms included, stipulate the execution of Clinical Trials. 